\title{
Staircase Dynamics of a Photonic Microwave Oscillator Based on a Laser Diode with Delayed Optoelectronic Feedback
}

\author{
Md Shariful Islam $\odot,{ }^{1,2,{ }^{*}}$ A.V. Kovalev $\odot,{ }^{3, \dagger}$ G. Coget, ${ }^{1}$ E.A. Viktorov, ${ }^{3, \grave{\dagger}}$ D.S. Citrin,,${ }^{1,2}$ and \\ A. Locquet $\circledast^{1,2, \S}$ \\ ${ }^{1}$ Georgia Tech-CNRS UMI 2958, Georgia Tech Lorraine, 2 Rue Marconi, 57070 Metz, France \\ ${ }^{2}$ School of Electrical and Computer Engineering, Georgia Institute of Technology, Atlanta, Georgia 30332-0250, \\ USA \\ ${ }^{3}$ ITMO University, Birzhevaya Liniya 14, 199034 Saint Petersburg, Russia
}

(Received 5 September 2019; revised manuscript received 14 May 2020; accepted 26 May 2020; published 16 June 2020)

\begin{abstract}
A laser diode subjected to optoelectronic feedback in which some light is converted to photocurrent that is fed back into the laser injection terminals can display periodic oscillations in its optical intensity. We demonstrate experimental and numerical evidence that, as the feedback level is varied, a stepwise change in the oscillation frequency manifests itself in the output optical intensity. These transitions in the dynamics can either correspond to an abrupt jump between two limit cycles, associated with a subcritical torus bifurcation of a limit cycle, or to a progressive switching mediated by an intermediate quasiperiodic state. Finally, when ramping the feedback level down, hysteresis is observed in the sequence of switching events between the limit cycles. Such devices are of interest for photonic microwave sources for wavelength-division multiplexed radio-over-fiber systems.
\end{abstract}

DOI: 10.1103/PhysRevApplied.13.064038

\section{INTRODUCTION}

Semiconductor laser diodes (LDs) subjected to optoelectronic (OE) feedback on their injection current $J$ have been a testbed for studying nonlinear dynamics in timedelayed systems for decades [1-4]. On the one hand, they provide an opportunity to study rich dynamical behaviors [5-7]; on the other, optoelectronic feedback systems have diverse applications ranging from microwave photonic generation [8,9] to controlled wideband frequency chirps [10] as well as in communications [11]. Optoelectronic oscillators with time delayed feedback and their applications are reviewed in Ref. [12].

Radio-over-fiber (ROF) is one such application of microwave photonics in which microwave-modulated optical signals are transmitted over fiber links [13]. ROF thus avoids expensive antennas, is not subject to losses of microwave waveguides or free-space propagation, and can provide access to areas where radio links might otherwise be infeasible. Specifically, wavelength-division multiplexed (WDM) systems can be used [14] to maximally exploit the optical bandwidth of fiber. Such WDM ROF systems clearly benefit from microwave-modulated optical

\footnotetext{
*mdshariful.islam@gatech.edu

†avkovalev@niuitmo.ru

¥evviktor@gmail.com

§alocquet@georgiatech-metz.fr
}

sources that can be tuned to the center microwave frequency of the relevant bands. Such frequency bands may be a few hundreds of $\mathrm{MHz}$ to a few $\mathrm{GHz}$ apart depending on the modulation of the center microwave frequency. This contribution explores a photonic microwave source based on a LD with OE feedback whose microwave frequency can be discretely tuned in steps on the hundred $\mathrm{MHz}$ to few $\mathrm{GHz}$ range. The device is based on a $\mathrm{LD}$ with optoelectronic feedback. For appropriate conditions of the feedback strength $\eta$, relaxation oscillations (ROs) become undamped resulting in a modulation of the output optical intensity $\mathcal{I}(t)$ in the $\mathrm{GHz}$ range. The RO frequency $f_{\mathrm{RO}}$ is modified by the nonlinear response of the gain medium as well as by the feedback, and the modulation frequency can be tuned in approximately $100-\mathrm{MHz}$ steps. The tuning is achieved based on the switching of the device dynamics between limit cycles that occur in certain regimes when the feedback strength is varied.

In retrospect, one of the earliest reports on the nonlinear dynamics of LDs with OE feedback is Ref. [1]. In a relatively simple setup, light output by the LD is incident on a photodiode (PD), which produces a current that is reinjected into the LD. References $[2,3]$ explore the situation theoretically, where various pulsing states, bistability, and chaos are predicted.

Subsequent works consider the distinction between negative $[6,15,16]$ and positive $[5,17]$ OE feedback. In the case of negative feedback, the fed-back current is subtracted 
from $J$ and is reported to be useful for tunable microwave [9] and optical short-pulse generation [18] by stabilizing the dynamical states. In this instance, the dynamics resulting from a locking between the RO frequency $f_{\mathrm{RO}}$ and the feedback-loop frequency $f_{\tau}$ (inverse of the delay time $\tau)$, and rational fractions of $f_{\mathrm{RO}}$ and $f_{\tau}$ participate in various dynamical states [6,19]. In most of these reports, a quasiperiodic (QP) route to chaos is observed. An exception is presented in Ref. [16] where a period-doubling route to chaos is observed, albeit for a ring laser. For positive feedback, the fed-back current from the OE loop is added back to $J$, which results in early pulsing states explained by a gain-switching mechanism [20]. The most common route to chaos with positive feedback is also QP [5]. Although the dynamics are not radically different from negative feedback, positive-feedback setups have been found to lack a few of the frequency-locked states that are common in negative feedback.

This study presents experimental and theoretical results for negative $\mathrm{OE}$ feedback showing discrete switching events between two dynamic states corresponding to stable limit cycles (LCs) as the feedback strength $\eta$ is gradually varied. These switching events are manifested in the frequency dynamics observed in the power spectrum of the photodetected optical intensity $\mathcal{I}(t)$, i.e., the rf spectrum. Although discrete switching events in the rf are well investigated for optical feedback and can correspond to jumps between two limit cycles located around the same externalcavity mode (ECM) [21-24] or different ECMs [25], the dynamics of OE feedback, and frequency switching in particular has been relatively sparsely explored. In Refs. [2123], rf switching with optical feedback is attributed to noise-induced hopping to a neighboring attractor separated by the feedback frequency around the same ECM. In Ref. [25], also with optical feedback, switching between LCs (also separated by $f_{\tau}$ ) is experimentally observed around the same ECM as well as two different ECMs as $\eta$ is varied, which is closer to the scenario reported here. To our knowledge none of the reports on OE feedback [5-7,9] have presented the analysis of switching between periodic states with distinct microwave frequencies.

Specifically, we focus on discrete transitions between limit cycles, manifested as stable periodic state with multi$\mathrm{GHz}$ oscillations in both the rf spectrum and in the optical modes. The rf spectra exhibit sharp peaks at distinct frequencies close to $f_{\mathrm{RO}}$, whereas the optical spectra present a number of equidistant lines separated by $f_{\mathrm{RO}}$ in optical frequency. We observe transitions between LCs separated in frequency by small multiples of $f_{\tau}$ that can either be abrupt or progressive as $\eta$ is ramped up. In the latter case of a progressive transition, a mediating quasiperiodic regime is involved. The optical spectrum however, shows shifting of the optical modes towards the solitary laser frequency at the switching events. These two types of transitions are still observed when $\eta$ is ramped down; however, the values of $\eta$ where a given switching event occur are lower, revealing hysteretic behavior. Of note, although the rf switching can correspond to a frequency jump of about $f_{\tau}$, as in the case of optical feedback, the underlying physics is found to differ significantly as no external-cavity modes exist in the case of optoelectronic feedback.

The paper is organized as follows. Section II presents the experimental setup; Sec. III describes the experimentally observed transitions between LCs; Sec. IV is devoted to the investigation of the results on the basis of a rate-equation model; finally, results are discussed and conclusions are drawn in Sec. V.

\section{EXPERIMENTAL SETUP}

A single-mode edge-emitting In-As/In-P quantum-dash (Qdash) distributed-feedback LD has been used for the experiment. The LD operating at $1550-\mathrm{nm}$ wavelength contains an active region stacked with six In-As Qdash layers embedded within a In-Ga-As-P quantum well and partitioned by In-Ga-As-P barriers. The internal cavity has a length of $500 \mu \mathrm{m}$, and the facets are antireflection coated. The threshold current is measured $J_{\text {th }} \sim 33 \mathrm{~mA}$ at room temperature. The experimental setup is depicted in Fig. 1 (see Ref. [9] for device and equipment details). The feedback loop is composed of optical free space (red), optical fiber (blue), and electronic (black) parts with total delay $(\tau)$ of $9.85 \mathrm{~ns}$. The electronic path that is composed of the SMA cable, photodetector, amplifiers, and microwave splitter contributes approximately $6.9 \mathrm{~ns}$ to the delay.

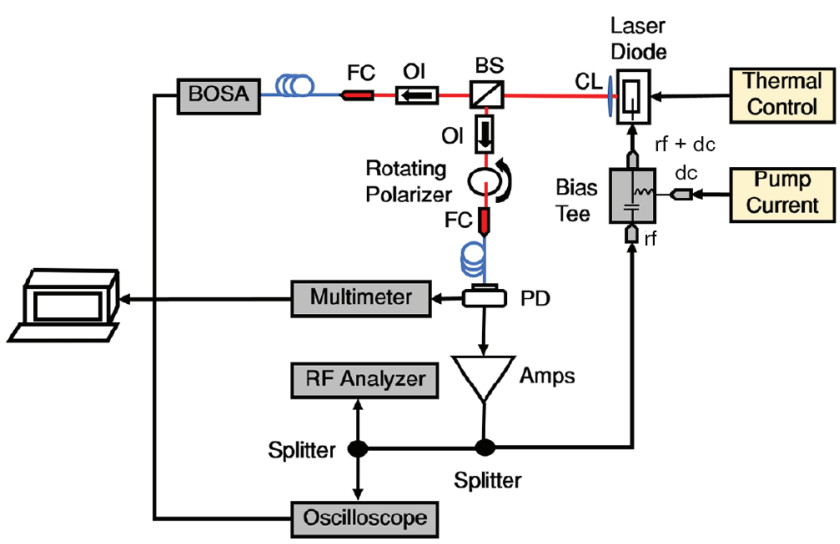

FIG. 1. Schematic diagram of experiment. The red line corresponds to optical free space, blue to optical fiber, and black to electrical cable. CL, collimation lens; BS, beam splitter; OI, optical isolator; FC, fiber coupler; PD, photodiode; Amps, amplifiers; BOSA, balanced optical spectrum analyzer. A 50:50 beam splitter is used to monitor the optical spectrum, whereas a 50:50 rf splitter is used to monitor simultaneously the signal with the oscilloscope (12-GHz bandwidth) and rf spectrum analyzer (26.5-GHz bandwidth). The multimeter provides a means to monitor the effective feedback level by measuring the PD bias voltage. 
A regulated current source drives the thermally stabilized LD. A linear polarizer (LP) mounted on a rotation stage is included in the optical path before the PD to tune $\eta$. The optical isolators (OIs) are introduced to improve the operating stability by preventing back reflections into the LD. The PD (12-GHz bandwidth) includes a low-noise amplifier that preamplifies the photodiode output before sending it to the cascade of amplifiers. The $18-\mathrm{dB}$ noninverting amplifier (Newport 1422-LF-20 GHz) and a 30-dB inverting amplifier (Microsemi UAOL30VM-30 $\mathrm{GHz}$ ) boost the signal to a level that can perturb the steady state of the laser. The output of the latter amplifier is directly added to $J$ using a bias tee (26.5-GHz bandwidth).

The experimental feedback level $\eta$ here is a relative measure of the feedback strength and cannot be directly compared to the theoretical $\eta$ used in the model presented in Sec. IV. The parameter $\eta$ is experimentally controlled through the linear polarizer; it is affected by the responsivity of the photodetector and the net gain of the components in the electronic loop. Conventionally, $\eta=1$ corresponds to full transmission by the linear polarizer. Rotating the LP allows a nonlinear control of the feedback level, since the attenuation introduced varies as the cosine square of the angle $(\phi)$ between the LP and the direction of polarization of the laser. Indeed, the effective feedback level is proportional to the quantity presented as $\eta\left(=\cos ^{2} \phi\right)$. For instance, full transmission (i.e., $\eta=1$ ) corresponds to $26 \%$, whereas $\eta=0.5$ represents only $8.4 \%$ of the rootmean-squared current fed back to the injection terminal as determined from the PD bias monitor.

The loop delay frequency is determined as $f_{\tau}=\tau^{-1}=$ $101 \mathrm{MHz}$. The lower cutoff frequency of the feedback loop is set by the $18-\mathrm{dB}$ amplifier, which is approximately 50 $\mathrm{MHz}$, whereas the upper cutoff is limited by the bandwidth of the 12-GHz PD.

\section{SEQUENCE OF DYNAMICAL REGIMES}

Figure 2 shows (a) the experimental bifurcation diagram (BD), (b) the rf spectra of $\mathcal{I}(t)$, and (c) the optical spectra of the laser obtained by and presented as a function of $\eta$ ramping up for $J \sim 2 J_{\text {th }}$. The $\mathrm{BD}$, representing the colormap for the histogram of the local extrema of $\mathcal{I}(t)$ at each $\eta$, reveals a sequence of dynamical regimes. Discrete changes in rf trending downward, occur at $\eta=0.52$, 0.82 , and 0.87 corresponding to switching between LCs. In the next paragraphs, we describe the sequence of events associated with these discrete changes in rf.

To begin with Fig. 2(a), the OE system shows a cw lasing for $\eta<0.47$; afterwards a $\mathrm{LC}\left(\mathrm{LC}_{1}\right)$ appears as a periodic modulation of $\mathcal{I}(t)$ with frequency $6.165 \mathrm{GHz}$ [Fig. 2(b) shows the rfs]. As a result of increasing $\eta$ further $(\eta \sim 0.52)$, the laser switches abruptly to another LC $\left(\mathrm{LC}_{2}\right)$ with a different dominant $\mathrm{rf}(6.067 \mathrm{GHz})$ separated by $f_{\tau}$. (a)
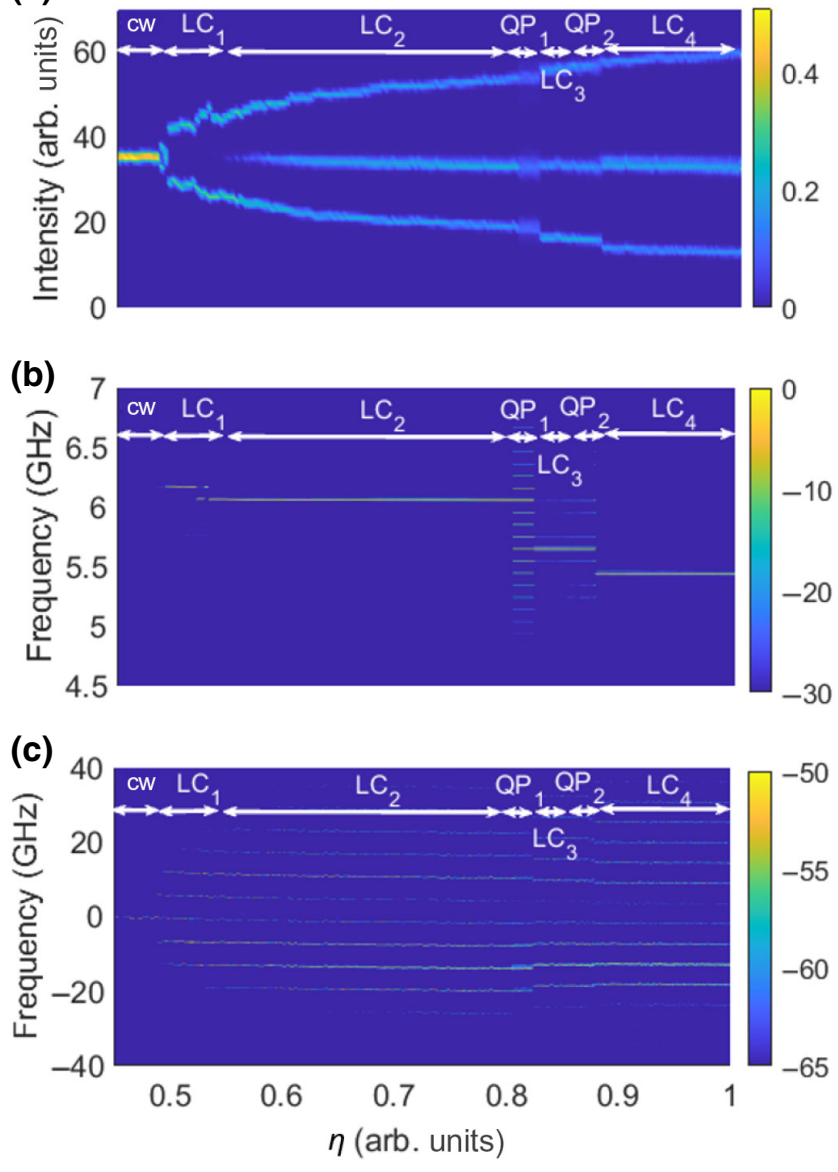

FIG. 2. (a) Experimental BD, (b) rf spectrum, and (c) optical spectrum with increasing $\eta$, on a logarithmic color scale. $\mathrm{cw}$, continuous waves; LC, limit cycle; QP, quasiperiodic.

The $\mathrm{LC}_{2}$ persists until $\eta \sim 0.80$, where another dynamical regime is reached, with the same main $\mathrm{rf}$ as in $\mathrm{LC}_{2}$, but numerous, strong, sidebands appear in the rf spectrum, corresponding to $\mathrm{QP}$ behavior $\left(\mathrm{QP}_{1}\right)$ as reported in Ref. [5]. Thereafter, at $\eta \sim 0.82$, we observe a jump to a different $\mathrm{LC}\left(\mathrm{LC}_{3}\right)$ with dominant rf $5.649 \mathrm{GHz}$. We refer to this jump as a progressive switching event, as the transition does not occur directly between $\mathrm{LC}$ regimes. After the jump, for even larger $\eta, \mathrm{LC}_{3}$ gives way to another mediating QP state $\left(\mathrm{QP}_{2}\right)$ and finally, for $\eta \sim 0.87, \mathrm{LC}_{4}$ with $\mathrm{rf}$ $5.442 \mathrm{GHz}$ is observed.

Meanwhile, the corresponding progression of optical lines, as shown in Fig. 2(c), is going to be addressed now. The optical frequency in the $\eta \rightarrow 0$ limit is the solitary laser frequency $f_{0}(\eta=0)$; for convenience, $f_{0}(0)$ is used to define the zero of the optical-frequency scale. As $\eta$ increases, additional optical lines appear, as is evident in Fig. 2(c). These lines occur at frequencies $f_{m}(\eta) \approx m f_{\mathrm{RO}}$, with $m$ an integer defining harmonics of $f_{\text {RO }}$.

Indeed, the jumps in the rf spectra towards some lower frequency can be related to the jumps in optical lines 
towards $f_{0}(0)$. Since the spectral separation between any two optical lines are $f_{\mathrm{RO}}$, with a downward jump in $\mathrm{rf}$ spectra, the optical lines recoordinate themselves with a similar jump multiplied by their respective harmonic positions [e.g., if $f_{1}(\eta)$ jumps by $f_{\tau}, f_{2}(\eta)$ evidently would have a jump of $2 f_{\tau}$ ]. Another point to be noted in Fig. 2(c), at $\eta=0.58$ the dominant optical frequency changes from $f_{-1}(\eta)$ to $f_{-2}(\eta)$ when the BD hints the start of a period doubling regime.

In summary, we see one abrupt switching at $\eta=0.52$ and two progressive switching at $\eta=0.82$ and 0.87 between two LCs in the LD dynamics. In the ensuing sections, we focus on these two different types of switching for detailed explanation and analysis, exploiting the simultaneous measurement of $\mathcal{I}(t)$, the rf, and optical spectra.

\section{A. Abrupt switching between two limit cycles}

We focus here on the jump from $\mathrm{LC}_{1}$ to $\mathrm{LC}_{2}$ at $\eta=0.52$. In order to elucidate the nature of this jump, Fig. 3 shows the $\mathrm{rf}$ and optical spectra just before $\left(\mathrm{LC}_{1}\right)$ and after $\left(\mathrm{LC}_{2}\right)$ with the corresponding time series for $\mathcal{I}(t)$ as the inset, which are approximately sinusoidal for both $\mathrm{LC}_{1}$ and $\mathrm{LC}_{2}$. The rf of oscillation switches abruptly from $6.165 \mathrm{GHz}$ for $\mathrm{LC}_{1}$ to $6.067 \mathrm{GHz}$ for $\mathrm{LC}_{2}$. The frequency difference of $98 \mathrm{MHz}$ is slightly less than $f_{\tau}$, which is similar to the case of optical feedback where the jumps in rf spectra are slightly smaller than $f_{\tau}$ [21]. In the optical spectra, we see a similar jump of the optical lines closer to the mode
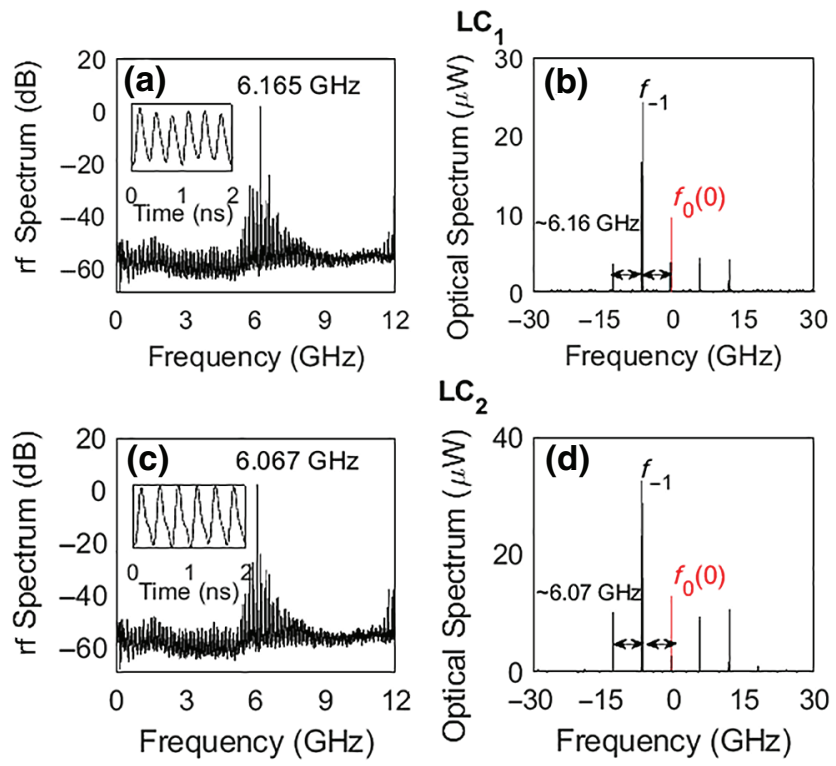

FIG. 3. Abrupt jump between LCs. (a),(b) $\eta=0.518\left(\mathrm{LC}_{1}\right)$, (c),(d) $\eta=0.525\left(\mathrm{LC}_{2}\right)$. (a),(c) rf spectra (inset: corresponding intensity time traces). (b),(d) Optical spectra. From (a),(c), a downward jump of $98 \mathrm{MHz}\left(\sim f_{\tau}\right)$ in rf oscillating frequency is observed between the two LCs. near $f_{0}(0)$; evidently, distant modes jump in bigger steps (depending on their location) to make the frequency separation between optical lines same as the new $f_{\text {RO }}$. We refer to this jump evidenced in the rf spectrum as an abrupt jump between two LCs.

\section{B. Progressive switching between two limit cycles}

In this section, we describe the two progressive jumps observed in Fig. 2. The first progressive jump occurs at $\eta \sim 0.82$. Figure 3 shows the rf and optical spectra before and after the jump. We notice a different type of transition from $\mathrm{LC}_{2}$ to $\mathrm{LC}_{3}$, as it involves mediating dynamics $\mathrm{QP}_{1}$. $\mathrm{QP}_{1}$ gives way to limit cycle $\mathrm{LC}_{3}$, whose frequency is smaller than that of $\mathrm{LC}_{2}$ by approximately $4 f_{\tau}$. Heuristically, the sidebands in the $\mathrm{QP}_{1}$ regime seem to favor the appearance of jumps in frequency to distant bifurcation branches, leading to frequency hops of more than $f_{\tau}$. Specifically, the fundamental frequency of $\mathrm{LC}_{3}(5.649$ $\mathrm{GHz}$ ) corresponds to the frequency of one of the sidebands of $\mathrm{QP}_{1}$. There appears to be a 1:15 resonance between the fast and slow frequencies of the $\mathrm{QP}_{1}$ dynamics, similar to the frequency-locked regimes reported in Ref. [19] by some of the co-authors. The locking is manifested in the time domain by the appearance of pulselike oscillation with a $100-\mathrm{MHz}$ repetition rate.

The optical spectra of the regimes involved $\left(\mathrm{LC}_{2}, \mathrm{QP}_{1}\right.$, $\mathrm{LC}_{3}$ ), reveal a series of optical lines separated by $6 \mathrm{GHz}$, before the jump, corresponding to the $\mathrm{rf}$ of $\mathrm{LC}_{2}$ and after the jump, optical lines are separated by $5.6 \mathrm{GHz}$. In addition, the optical lines $f_{m}(\eta)$ jump closer to $f_{0}(\eta)$ by $m$ times $4 f_{\tau}$. For the dominant optical line $f_{-2}(\eta)$, it jumps towards $f_{0}(\eta)$ by approximately $2 \times 4 f_{\tau}$ since it is the second harmonic of $f_{0}(\eta)$. Besides that, some of the optical lines of $\mathrm{QP}_{1}$ have a multipeaked appearance [inset of Fig. 4(b)] that evidence the emergence of additional optical frequencies.

The last jump at $\eta \sim 0.87$, is shown in Fig. 5. The $\mathrm{rf}$ spectrum and its intensity time series [Fig. 5(a), inset] highlight the quasiperiodic $\left(\mathrm{QP}_{2}\right)$ nature of the dynamics before the jump, with a main frequency at $5.649 \mathrm{GHz}$ and sidebands separated by $100 \mathrm{MHz}$. After the jump, limitcycle dynamics $\mathrm{LC}_{4}$ is reached, with a dominant frequency of $5.442 \mathrm{GHz}$. We thus observe again a jump of $207 \mathrm{MHz}$ in rf, corresponding to approximately $2 f_{\tau}$. To be noted, $\mathrm{QP}_{1}$ in the previous progressive jump appears instantly after $\mathrm{LC}_{2}$, whereas $\mathrm{QP}_{2}$ evolves gradually from $\mathrm{LC}_{3}$ as we keep increasing $\eta$. Limit cycle $\mathrm{LC}_{3}$ develops increasingly more sidebands, turning into a quasiperiodic state $\mathrm{QP}_{2}$, before finally shifting by approximately $2 f_{\tau}$ [to the less prominent second major sidebands, indicated by a red arrow in Fig. 5(a)] and ended up yielding an ideal $\mathrm{LC}\left(\mathrm{LC}_{4}\right)$ all of a sudden.

Similar to the other bifurcations, the optical spectra before and after the jump [Fig. 5(d)] show a series of optical lines and $f_{-1}(\eta)$ and $f_{1}(\eta)$ that are both separated by 

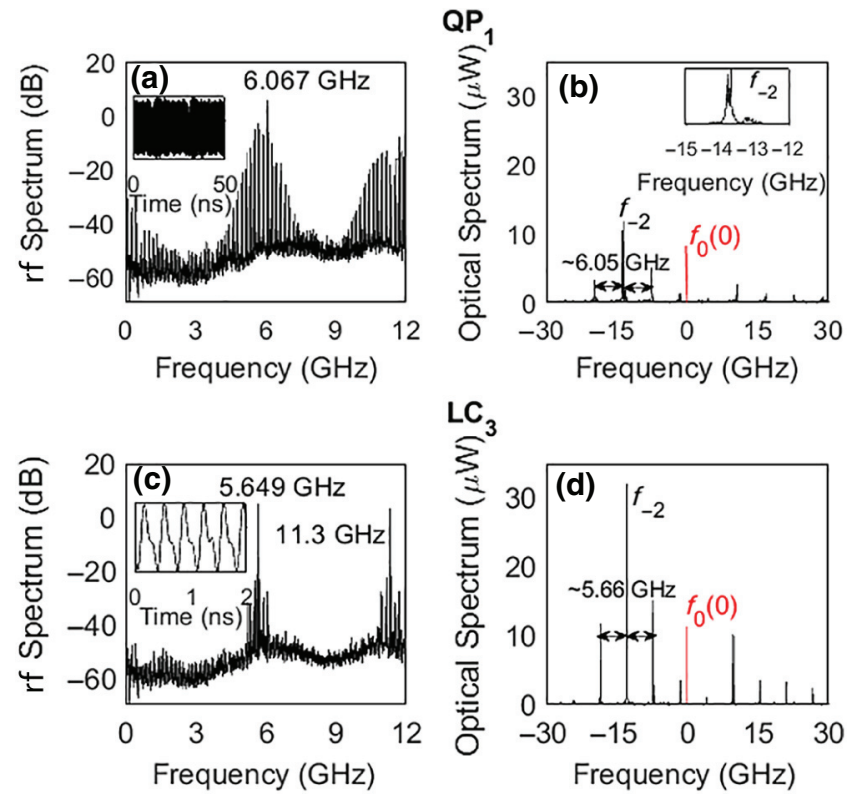

FIG. 4. First progressive jump. (a),(b) $\eta \sim 0.81\left(\mathrm{QP}_{1}\right)$, (c),(d) $\eta \sim 0.824\left(\mathrm{LC}_{3}\right)$. (a),(c) rf spectra (inset: corresponding intensity time traces). (b),(d) Optical spectra. From (a),(c), a downward jump of $418 \mathrm{MHz}\left(\sim 4 f_{\tau}\right)$ in rf oscillating frequency is observed between a QP and a LC. The inset in (a) is presented for a longer range of time to show the presence of secondary oscillation in the time series. The inset in (b) shows an enlarged peak at $f_{-2}$ evidencing the emergence of additional optical frequencies.

approximately $5.67 \mathrm{GHz}$ from $f_{0}(\eta)$ before the jump scale down to $5.44 \mathrm{GHz}$ after the jump. All the optical lines jump closer to $f_{0}(\eta)$ by a multiple of $207 \mathrm{MHz}$ depending on their harmonic positions as before.

\section{Hysteresis and robustness}

We now discuss the presence of hysteresis in reference to the switches and the robustness of our observation in this section. First, Figs. 6(a) and 6(b) show the BD of $\mathcal{I}(t)$ and the rf spectrum ramping $\eta$ down adiabatically, in contrast to what is seen in Figs. 2(a) and 2(b) in which $\eta$ is ramped up. The frequencies of LCs seen in the rf spectra are the same as those in Fig. 2 but the jumps tend to occur at different values of $\eta$.

Moreover, when $\eta$ is ramped down, the first jump appearing at $\eta=0.73$ is an abrupt jump between two LCs; the second jump appearing at $\eta=0.62$ is a progressive jump involving a strong mediating QP state; and the last jump appearing at $\eta=0.55$ is an abrupt jump between two LCs as well. Therefore, we observe two abrupt jumps and one progressive jump ramping $\eta$ down, whereas there are one abrupt and two progressive jumps in ramping $\eta$ up.

In addition, the BD also shows a qualitatively different trend when $\eta$ is ramped down. Indeed, when ramping $\eta$ up the peak magnitude of $\mathcal{I}(t)$ increases steadily, then a sharp increase in magnitude occurs parallel to the rf spectral
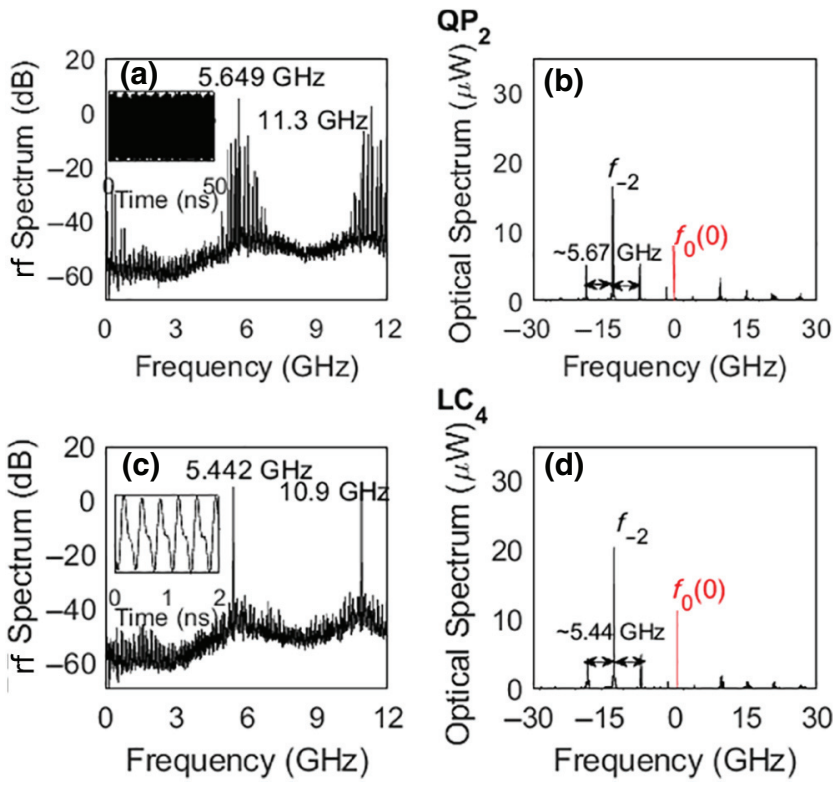

FIG. 5. Second progressive jump. (a),(b) $\eta \sim 0.874\left(\mathrm{QP}_{2}\right)$, (c),(d) $\eta \sim 0.876\left(\mathrm{LC}_{4}\right)$. (a), (c) rf spectra (inset: corresponding intensity time traces). (b),(d) Optical spectra. From (a),(c), a downward jump of $207 \mathrm{MHz}\left(\sim 2 f_{\tau}\right)$ in rf oscillating frequency is observed between a QP and a LC. Similar to the previous figure the inset in (a) is presented for a longer range of time to show the presence of secondary oscillation in the time series.

jumps; when ramping $\eta$ in the opposite direction, however, the magnitude gradually decreases to a minima before jumping up abruptly to a higher magnitude for all of the switches [compare Figs. 2(a) and 6(a)].
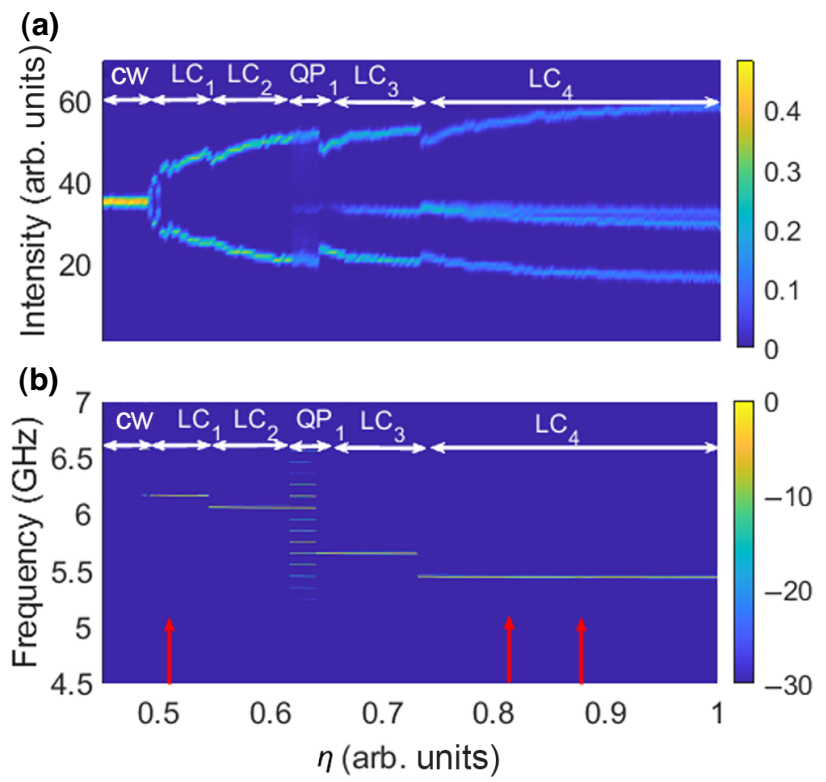

FIG. 6. (a) Reverse BD where $\eta$ is ramped down from the maximum. (b) Corresponding rf spectrum. Red arrows indicate the jumps in the ramping up direction of $\eta$. 
We also verify experimentally the robustness of the results for different pump currents $J$ in the range 50 to 70 $\mathrm{mA}$ and find similar sequences of bifurcations, including the jumps discussed above. Of note, while abrupt transitions can either be a jump in frequency by small multiples of $f_{\tau}$, progressive transitions are always found to imply frequency jumps of nonunit multiples of $f_{\tau}$.

\section{LASER MODEL}

Dynamical studies of delayed feedback systems have been investigated within the Eckhaus instability framework [26-29] as well as a rate equation model [21-24]. We employ a rate-equation model for the laser with optoelectronic filtered feedback, which allows only high-frequency signals to pass through the feedback loop. The model is based on that in Ref. [30], and consists of the following equations for the normalized optical intensity $I(t)$, the carrier density $N(t)$, and the high-pass filtered intensity signal $I_{F}(t)$. They are given by

$$
\begin{gathered}
\dot{I}(t)=2 N(t) I(t) \\
\dot{I}_{F}(t)=-\tau_{F}^{-1} I_{F}(t)+\dot{I}(t) \\
\varepsilon^{-1} \dot{N}(t)=P-\eta I_{F}(t-\tau)-N(t)-[1+2 N(t)] I(t) .
\end{gathered}
$$

The dot means differentiation with respect to $t=\hat{t} / \tau_{\mathrm{ph}}$ where $\hat{t}$ is time, and $\tau_{\mathrm{ph}}$ is the photon lifetime. $P=$ $J / J_{\text {th }}-1$ is the pump above the threshold parameter; $\eta$ is the feedback strength (for consistency with experimental description, $\eta>0$ is for negative feedback, and $\eta<0$ is for positive feedback); $\tau$ is the feedback delay; $\varepsilon$ is the ratio of the carrier and photon lifetimes; $\tau_{F}{ }^{-1}$ is the low-cut (high-pass) filter bandwidth.

We determine the characteristic equation from the linearized Eqs. (1)-(3) for the nonzero intensity steady state $\left(I=P, I_{F}=0, N=0\right)$ and find

$$
\left(1+\tau_{F} \lambda\right)\left\{\lambda^{2}+\varepsilon[\lambda+2 P(1+\lambda)]\right\}-2 \varepsilon \eta \tau_{F} P \lambda e^{-\tau \lambda}=0 .
$$

The stability boundaries correspond to the Hopf bifurcation condition $\lambda= \pm i \omega_{H}$, and the Hopf frequency $\omega_{H}$ can be determined from the transcendental equation, which is given by

$\arctan \frac{\left[1+2 P\left(1+\tau_{F}\right)\right] \varepsilon \omega_{H}-\tau_{F} \omega_{H}^{3}}{\left[1+\varepsilon \tau_{F}(1+2 P)\right] \omega_{H}^{2}-2 \varepsilon P}=\omega_{H} \tau+\frac{\pi}{2}-\pi M$,

where $M$ is a non-negative integer number and indicates the existence of multiple Hopf bifurcation branches in the dynamics. $M$ is odd for the negative feedback $(\eta>0)$, and is even for the positive feedback $(\eta<0)$.

The Hopf bifurcation point $\eta_{H}$ can be determined analytically,

$$
\eta_{H}=\frac{\varepsilon+2 \varepsilon P\left(1+\tau_{F}\right)-\tau_{F} \omega_{H}^{2}}{2 \varepsilon \tau_{F} P \cos \omega_{H} \tau} .
$$

The discretization of the frequencies in Eq. (5) is parity asymmetric with relation to the feedback sign since different resonant conditions are formed by the parity of $M$ in Eq. (5), and the $\cos \omega_{H} \tau$ term in Eq. (6).

In the long delay limit $\tau \gg 1$ and when the filter cutoff frequency is smaller than the RO frequency, i.e., $\omega_{\mathrm{RO}} \tau_{F} \gg$ 1 , where $\omega_{\mathrm{RO}}=\frac{1}{2} \sqrt{8 \varepsilon P-\varepsilon^{2}(1+2 P)^{2}}$ is the RO angular frequency of the free-running laser $(\eta=0)$ [30], Eqs. (5) and (6) can be approximated, leading to the following expressions:

$$
\begin{gathered}
\omega_{H}=\sqrt{\omega_{\mathrm{RO}}^{2}-\gamma_{\mathrm{RO}}^{2}}, \\
\eta_{H}= \pm \frac{\gamma_{\mathrm{RO}} \omega_{\mathrm{RO}}}{\varepsilon P},
\end{gathered}
$$

where $\gamma_{\mathrm{RO}}=\frac{1}{2} \varepsilon(1+2 P)$ is RO damping rate. Using Eqs. (7) and (8) and knowing the frequency of the first limit cycle it is possible to easily determine the value of the ratio of the carrier and photon lifetimes $\varepsilon$, and we estimate the following parameter values for the system: $\varepsilon=0.095, \tau=1000, \tau_{F}=2000$. It is worth mentioning that the bifurcation border in this limit is symmetric with respect to the sign of $\eta$, which is not the case when the filtering is initially absent from the system [i.e., $I_{F}(t)=I(t)$ ] because then the steady-state intensity depends on $\eta$, and reads $I=P /(1+\eta)$.

Figure 7 shows the Hopf bifurcation curves in $(\eta, P)$ plane obtained by solving Eqs. (5) and (6) within the range relevant to the experimental parameters, and the bifurcations of the steady state at the value of pump about twice exceeding the threshold $(P=0.945)$. This steady state undergoes a sequence of the bifurcations each leading to the appearance of a limit cycle, which we discuss further.

We analyze the stability of the limit cycles arising from the Hopf bifurcations in Fig. 7 using the numerical continuation package DDE-Biftool [31] (see Fig. 8). The limit cycles originating from the Hopf bifurcations having frequencies higher than the frequency of the first Hopf bifurcation are ever unstable for the full range of $\eta$ variation while the limit cycles arising from the Hopf bifurcations with lower frequencies become eventually stable. The limit cycles stabilize (destabilize) through torus bifurcations indicated in Fig. 8 by blue circles. This forms the wide range of multistability, and hysteretic effects under 
(a)

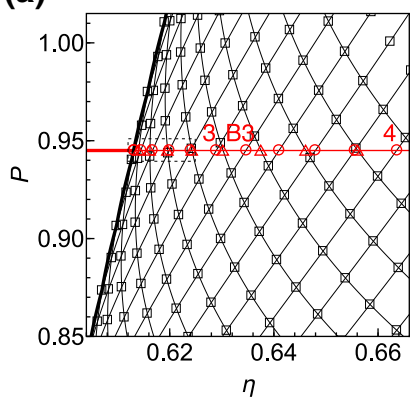

(b)

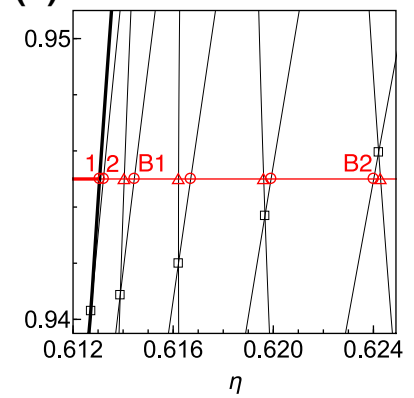

FIG. 7. Hopf bifurcation boundaries (black lines) in the $(\eta, P)$ parameter plane calculated from Eqs. (5) and (6). (b) The enlarged region delimited by the dashed rectangle in (a). Bold lines define the first bifurcation of the steady state. The thick (thin) red line is the stable (unstable) cw solution at $P=0.945$. The red circles (triangles) are the Hopf bifurcation points leading to eventually stable (ever unstable) limit cycles. The labels (1-4, B1-B4) correspond to the Hopf points leading to the limit cycles observed in numerical integration, and shown in Fig. 9. The other parameters are $\varepsilon=0.095, \tau=1000, \tau_{F}=2000$.

cyclic $\eta$ as shown experimentally. The mechanism responsible for stabilization (destabilization) of the limit cycles as the feedback parameter $\eta$ increases (decreases) is similar to the Eckhaus instability (see the Appendix for more detail).

To reproduce the experimental situation we directly integrate Eqs. (1)-(3) using the fourth-order Runge-Kutta method. Parameter $\eta$ is varied in a stepwise manner, with a step change $\delta_{\eta}=5 \times 10^{-4}$ every $4000 \tau$. The integration
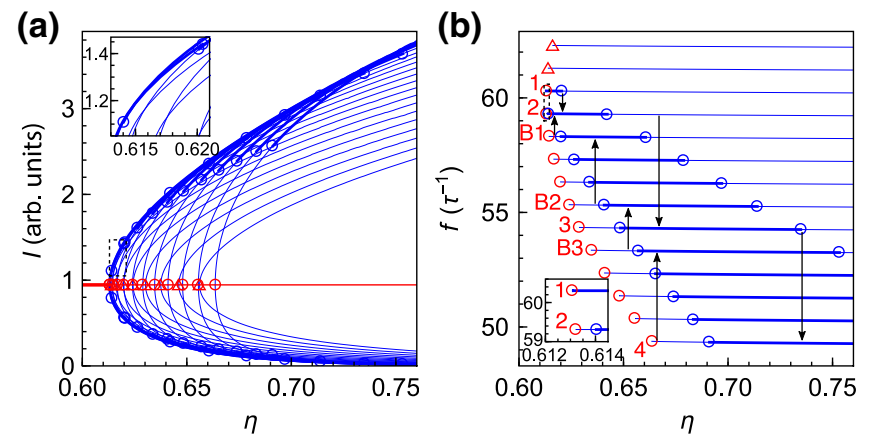

FIG. 8. Bifurcation diagrams showing the maxima and minima of intensity (a) and frequency (b) of limit cycles arising from the Hopf bifurcations of steady state at $P=0.945$ shown in Fig. 7. Thick (thin) blue lines are stable (unstable) limit cycles, and blue circles are torus bifurcations. The insets demonstrate enlarged areas delimited by dashed lines in the corresponding figures. The upward (downward) arrows in (b) show the transitions for $\eta$ increase (decrease) during numerical integration as demonstrated in Fig. 9. For simplicity, the Hopf bifurcations lying beyond the $\eta$ range in Fig. 7, and corresponding limit cycles are not shown. The other notations and parameters are the same as in Fig. 7. time step is fixed to $\tau / 5000$. A white-noise term with amplitude $10^{-5}\left(10^{-4}\right)$ is added to Eq. (1) [Eq. (3)] to model the spontaneous emission (feedback parasitic variations).

We follow the bifurcation sequence with increasing $\eta$ as shown in Fig. 9. Similar to the experiment, the $\mathrm{cw}$ state undergoes a supercritical Hopf bifurcation resulting in a small amplitude stable limit cycle $\mathrm{LC}_{1}$ with frequency approximately $60 \tau^{-1}$. The cycle grows in amplitude with $\eta$ increase, and undergoes an abrupt transition to a limit cycle $\mathrm{LC}_{2}$ having the frequency approximately $59 \tau^{-1} . \mathrm{LC}_{1}$ and $\mathrm{LC}_{2}$ are, therefore, separated by the feedback frequency $\tau^{-1}$ as is experimentally observed. In turn, $\mathrm{LC}_{2}$ develops into a quasiperiodic state $\mathrm{QP}_{1}$ through a supercritical bifurcation, and eventually collapses to the limit cycle $\mathrm{LC}_{3}$ with the frequency approximately $54 \tau^{-1}$. Therefore, $\mathrm{QP}_{1}$ is mediating transition from $\mathrm{LC}_{2}$ and $\mathrm{LC}_{3}$, which are separated by approximately $5 \tau^{-1}$, again very similar to the experiment. Finally, $\mathrm{LC}_{3}$ abruptly switches to the limit cycle $\mathrm{LC}_{4}$ with the frequency approximately $49 \tau^{-1}$.

Changing $\eta$ in the opposite direction, we find a bifurcation sequence, which is more complicated and indicates a hysteresis effect. The system follows the prolongated branch of the limit cycle $\mathrm{LC}_{4}$ until a transition to a quasiperiodic state $\mathrm{QP}_{\mathrm{B} 4}$. It then follows a sequence of progressive switching between limit cycles $\mathrm{LC}_{\mathrm{B} 3} \rightarrow$ $\mathrm{LC}_{\mathrm{B} 2}, \mathrm{LC}_{\mathrm{B} 2} \rightarrow \mathrm{LC}_{\mathrm{B} 1}, \mathrm{LC}_{\mathrm{B} 1} \rightarrow \mathrm{LC}_{2}$ intermediated by the quasiperiodic states $\mathrm{QP}_{\mathrm{B} 3}, \mathrm{QP}_{\mathrm{B} 2}$, and $\mathrm{QP}_{\mathrm{B} 1}$ correspondingly. The transition ends with the limit cycle $\mathrm{LC}_{2}$ followed by a steady-state solution. As seen in Fig. 8, the limit cycle $\mathrm{LC}_{2}$ has a very narrow region of instability between its origin and the stabilizing torus point. As far as the system is drastically slowing down near the Hopf bifurcation points, this is the reason why we observe transition from $\mathrm{LC}_{2}$ to the steady state instead of $\mathrm{LC}_{1}$ for $\eta$ decrease in Fig. 9.

The performed bifurcation analysis allowed us to identify and to classify multiple periodic and quasiperiodic solutions, which are dense in space. It is worth emphasizing here that switching between the limit cycles appears through torus bifurcations, which either form mediating stable quasiperiodic state or lead to abrupt transition to a limit cycle. Both types of transitions we experimentally confirm. The stability analysis also explains why the experimental switching happens to the limit cycles with lower frequencies, which become eventually stable with the feedback strength increase as consistent with the Eckhaus mechanism. The rf staircase scenario seen ramping $\eta$ down fully corresponds to the major experimental features. However, one notes that the middle traces of the experimental intensity of some limit-cycle regions appearing in the bifurcation diagrams of Figs. 2 and 6 are not present in Fig. 9. This difference might be attributed to a number of factors such as the way filtering is modeled, nonlinear effects such as gain compression, and specificities of 

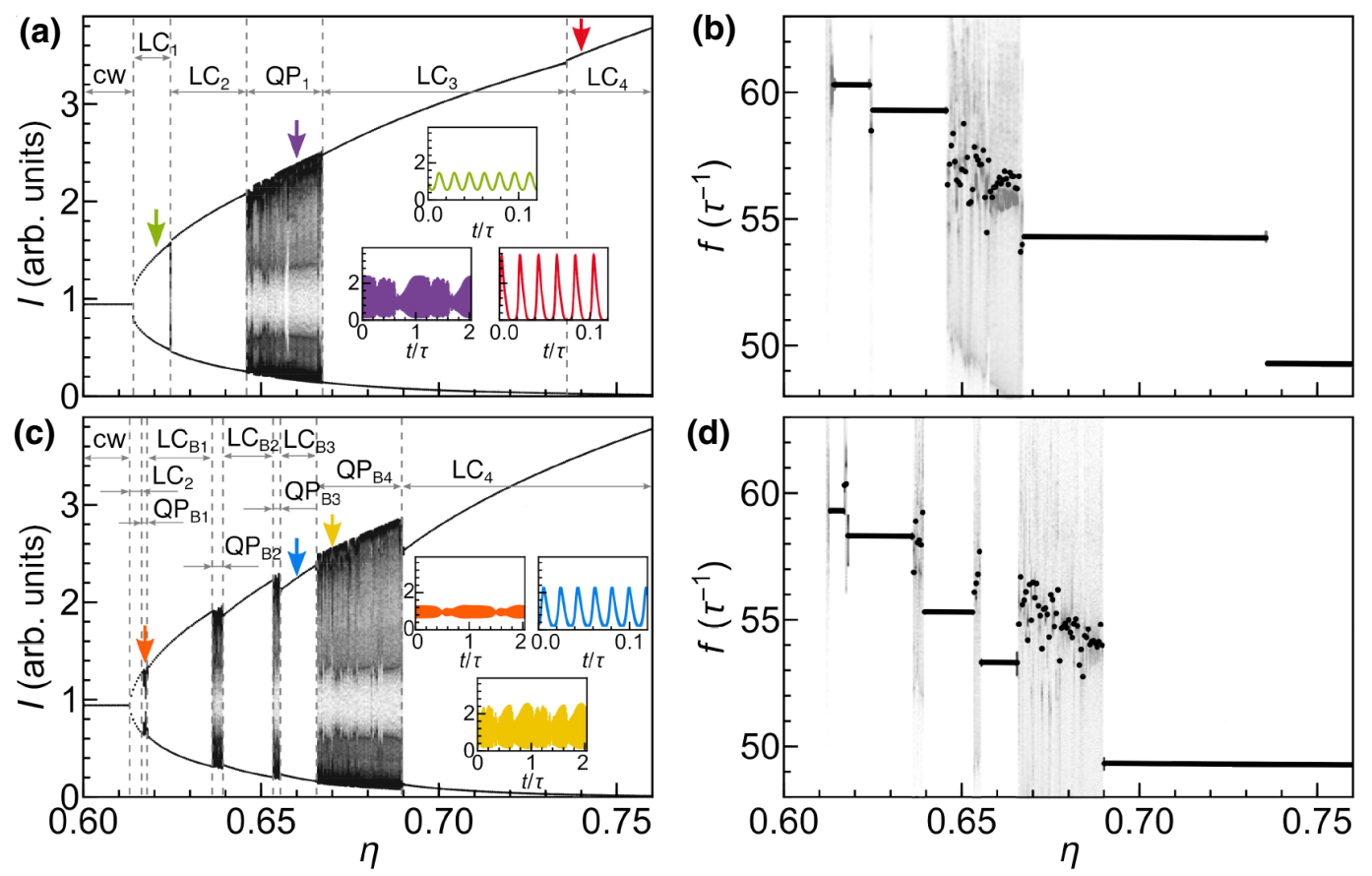

FIG. 9. Numerical bifurcation diagram obtained by integration of Eqs. (1)-(3) when $\eta$ is increased (a),(b) and decreased (c),(d). (a),(c) Local extrema of intensity $I$, and (b),(d) the frequency $f$ defined as the inverse of time intervals between two adjacent intensity maxima, where bold dots stand for the average values. cw, steady state; LC, limit cycle; QP, quasiperiodic state. The insets in (a),(c) show the exemplary intensity time traces at $\eta$ values given by the corresponding colored arrows: green, $\mathrm{LC}_{1}$ at $\eta=0.621 ; \mathrm{purple}, \mathrm{QP}_{1}$ at $\eta=0.66$; red, $\mathrm{LC}_{4}$ at $\eta=0.74$; orange, $\mathrm{QP}_{\mathrm{B} 1}$ at $\eta=0.6175$; blue, $\mathrm{LC}_{\mathrm{B} 3}$ at $\eta=0.66$; yellow, $\mathrm{QP}_{\mathrm{B} 4}$ at $\eta=0.67$.

the quantum-dash medium that are not included in the model. Nevertheless, the overall one-parameter bifurcation scenario is in excellent qualitative agreement with the experiment, showing the same characteristic dynamics, abrupt and progressive switching between limit cycles as well as the hysteretic behavior.

\section{DISCUSSION AND CONCLUSION}

The nonlinear dynamics of a laser diode with timedelayed optoelectronic feedback are explored with a focus on switching events between limit cycles. These are manifested in a change of the dominant frequency of $\mathcal{I}(t)$. One possible type of switching is abrupt and corresponds to a subcritical torus bifurcation of a limit cycle. Of note, similar abrupt switches between limit cycles have been observed in lasers subjected to optical feedback, and can involve, in this case, either different limit cycles born from the same external-cavity modes or limit cycles from different neighboring, modes. The switching between microwave frequencies in steps in the $100 \mathrm{MHz}$ range (shorter feedback times are expected to result in larger frequency steps) is of interest to produce photonic microwave sources for WDM ROF systems.

A progressive switching between two limit cycles is also observed in a certain range of $\eta$. This transition involves a mediating quasiperiodic regime that bridges limit-cycle branches. In all scenarios the frequency of limit cycles decrease when $\eta$ is increased. Hysteresis is observed in the modulation frequency as the feedback strength $\eta$ is ramped up or down. The possibility to control discrete switching between microwave frequencies in the $\mathrm{GHz}$ range could find applications in the field of microwave photonics.

Although we report discrete tunability from $5.44 \mathrm{GHz}$ to $6.17 \mathrm{GHz}$ in $100-\mathrm{MHz}$ steps as a function of feedback strength, the number of switching events and the range of LC dynamics are observed to increase at higher $J$. The proposed photonics MW generator is based on readily available off-the-shelf telecommunications components and is more compact compared with arrangements based on optical injection [32-34]. The range of tunability can be further improved by choosing appropriate lasers (e.g., vertical-cavity surface-emitting lasers), which may have $f_{\mathrm{RO}}$ of several tens of $\mathrm{GHz}$ as demonstrated in Ref. [32] using dual-beam orthogonal optical injection. The system performance can also be improved by employing lownoise electronic components (e.g., amplifiers). With this in mind, we point to our recent report [9], where utilization of optoelectronic feedback enabled sub-ps jitter and typical phase noise in the range of $-107 \mathrm{dBc} / \mathrm{Hz}$ at $10-\mathrm{kHz}$ offset. A similar approach could be used to improve the phase-noise characteristics of the present oscillator. That said, the current work is not focused on designing an ultrastable photonics MW source, but rather on the discrete 
switching events between limit cycles exhibiting dominant frequencies separated by a distinct frequency. Exploiting this phenomenon is of potential interest for future discretely tunable photonic microwave sources that can be used in multichannel communications.

\section{ACKNOWLEDGMENTS}

The work of A.V.K. and E.A.V. was supported by the Ministry of Education and Science of the Russian Federation (research project No. 2019-1442). A.V.K. and E.A.V. acknowledge the Government of the Russian Federation (Grant 08-08). A.L., D.S.C., G.C., and M.S.I. acknowledge the financial support of the Conseil Régional Grand Est and of the Fonds Européen de Développement Régional (FEDER).

\section{APPENDIX: ECKHAUS INSTABILITY}

Eckhaus instability refers to the generic scenario observed in spatially extended and delayed dynamic systems when multiple periodic regimes coexist, and their stability depends on the frequency [35]. The general framework used to explore this scenario extends from photonics, ecological, and neural-network-based instances, and is based on analysis of multiple time scales in the vicinity of a Hopf bifurcation point as was demonstrated for a delayed system in Ref. [26]. Figure 10 illustrates that the observed sequence of torus bifurcations leading to the stabilization

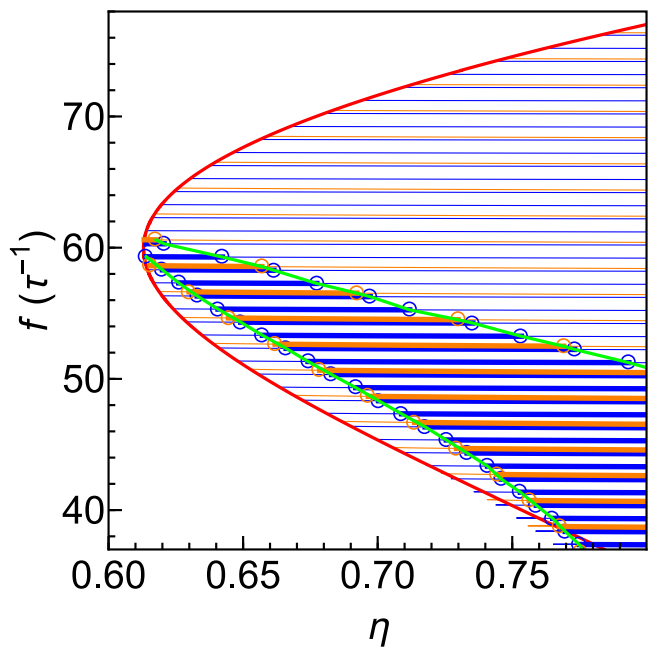

FIG. 10. Bifurcation diagrams obtained by numerical continuation for two different values of delay $\tau: 1000$ (blue) and 500 (orange). The vertical axis is given in units of $\tau^{-1}=0.001$ for consistency with Fig. 8. The red line is the Hopf bifurcation line given by Eq. (A1). Thick (thin) blue and orange lines correspond to stable (unstable) limit cycles, and the circles correspond to torus bifurcations. Green lines delimit the region of stable periodic solutions. Periodic branches with lower frequencies are born from subcritical Hopf bifurcations and extend beyond the red line. The other parameters are the same as in Figs. 7 and 8. of the limit cycles, and corresponding hysteretic behavior, when the feedback strength $\eta$ is decreased, are consistent with this generic mechanism.

Figure 10 shows an extended bifurcation diagram from Fig. 8(b) complemented with a bifurcation diagram calculated for a smaller delay value $\tau=500$, which can still be considered large for the parameter range under consideration. The red line shows a continuity of the Hopf frequency $\omega_{H}$ described by

$$
\eta=\frac{\sqrt{\omega_{H}^{4}-4 \varepsilon P \omega_{H}^{2}+\varepsilon^{2}\left[4 P^{2}+\omega_{H}^{2}(2 P+1)^{2}\right]}}{2 \varepsilon P},
$$

which is derived from Eqs. (5) and (6) under the assumption of $\omega_{\mathrm{RO}} \tau_{f} \gg 1$.

The green lines in Fig. 10 connect the torus bifurcation points that lead to stabilization or destabilization of the limit cycles, and approximate well the Eckhaus curve similar to those demonstrated in Refs. [26] and [35].

[1] G. Giacomelli, M. Calzavara, and F. Arecchi, Instabilities in a semiconductor laser with delayed optoelectronic feedback, Opt. Commun. 74, 97 (1989).

[2] C.-H. Lee and S.-Y. Shin, Self-pulsing, spectral bistability, and chaos in a semiconductor laser diode with optoelectronic feedback, Appl. Phys. Lett. 62, 922 (1993).

[3] E. Grigorieva, H. Haken, and S. Kaschenko, Theory of quasiperiodicity in model of lasers with delayed optoelectronic feedback, Opt. Commun. 165, 279 (1999).

[4] S. Shahin, F. Vallini, F. Monifi, M. Rabinovich, and Y. Fainman, Heteroclinic dynamics of coupled semiconductor lasers with optoelectronic feedback, Opt. Lett. 41, 5238 (2016).

[5] S. Tang and J. Liu, Chaotic pulsing and quasi-periodic route to chaos in a semiconductor laser with delayed optoelectronic feedback, IEEE J. Quantum Electron. 37, 329 (2001).

[6] F. Lin and J. Liu, Harmonic frequency locking in a semiconductor laser with delayed negative optoelectronic feedback, Appl. Phys. Lett. 81, 3128 (2002).

[7] F.-Y. Lin and J.-M. Liu, Nonlinear dynamics of a semiconductor laser with delayed negative optoelectronic feedback, IEEE J. Quantum Electron. 39, 562 (2003).

[8] X. S. Yao and L. Maleki, High frequency optical subcarrier generator, Electron. Lett. 30, 1525 (1994).

[9] M. J. Wishon, D. Choi, T. Niebur, N. Webster, Y. K. Chembo, E. A. Viktorov, D. Citrin, and A. Locquet, Lownoise $\mathrm{x}$-band tunable microwave generator based on a semiconductor laser with feedback, IEEE Photonics Technol. Lett. 30, 1597 (2018).

[10] N. Satyan, A. Vasilyev, G. Rakuljic, V. Leyva, and A. Yariv, Precise control of broadband frequency chirps using optoelectronic feedback, Opt. Express 17, 15991 (2009).

[11] H. D. Abarbanel, M. B. Kennel, L. Illing, S. Tang, H. Chen, and J. Liu, Synchronization and communication 
using semiconductor lasers with optoelectronic feedback, IEEE J. Quantum Electron. 37, 1301 (2001).

[12] Y. K. Chembo, D. Brunner, M. Jacquot, and L. Larger, Optoelectronic oscillators with time-delayed feedback, Rev. Mod. Phys. 91, 35006 (2019).

[13] G. Chang and P. Peng, in 2018 23rd Opto-Electronics and Communications Conference (OECC) (IEEE, Jeju Island, Korea (South), 2018), p. 1.

[14] Y. Zhu, Y. Wu, H. Xu, C. Browning, L. P. Barry, and Y. Yu, Experimental demonstration of a WDM-RoF based mobile fronthaul with f-OFDM signals by using directly modulated 3s-DBR laser, J. Lightwave Technol. 37, 3875 (2019).

[15] F. Lin and J. Liu, Nonlinear dynamical characteristics of an optically injected semiconductor laser subject to optoelectronic feedback, Opt. Commun. 221, 173 (2003).

[16] S. T. Kingni, G. Van der Sande, I. V. Ermakov, and J. Danckaert, Theoretical analysis of semiconductor ring lasers with short and long time-delayed optoelectronic and incoherent feedback, Opt. Commun. 341, 147 (2015).

[17] Y.-Y. Xie, H.-J. Che, W.-L. Zhao, Y.-X. Huang, W.-H. $\mathrm{Xu}, \mathrm{X} . \mathrm{Li}, \mathrm{Q}$. Kan, and J.-C. Li, Dynamics of 1550-nm VCSELs with positive optoelectronic feedback: Theory and experiments, IEEE Photonics J. 6, 1 (2014).

[18] C.-H. Lee, S.-Y. Shin, and S.-Y. Lee, Optical short-pulse generation using diode lasers with negative optoelectronic feedback, Opt. Lett. 13, 464 (1988).

[19] A. V. Kovalev, M. S. Islam, A. Locquet, D. S. Citrin, E. A. Viktorov, and T. Erneux, Resonances between fundamental frequencies for lasers with large delayed feedbacks, Phys. Rev. E 99, 062219 (2019).

[20] T. Damen and M. Duguay, Optoelectronic regenerative pulser, Electron. Lett. 16, 166 (1980).

[21] J. Mørk, J. Mark, and B. Tromborg, Route to Chaos and Competition between Relaxation Oscillations for a Semiconductor Laser with Optical Feedback, Phys. Rev. Lett. 65, 1999 (1990).

[22] J. Mørk, B. Tromborg, and J. Mark, Chaos in semiconductor lasers with optical feedback: Theory and experiment, IEEE J. Quantum Electron. 28, 93 (1992).

[23] A. Ritter and H. Haug, Theory of the bistable limit cycle behavior of laser diodes induced by weak optical feedback, IEEE J. Quantum Electron. 29, 1064 (1993).
[24] D. Pieroux, T. Erneux, T. Luzyanina, and K. Engelborghs, Interacting pairs of periodic solutions lead to tori in lasers subject to delayed feedback, Phys. Rev. E 63, 036211 (2001).

[25] B. Kim, A. Locquet, D. Choi, and D. S. Citrin, Experimental route to chaos of an external-cavity semiconductor laser, Phys. Rev. A 91, 061802(R) (2015).

[26] M. Wolfrum and S. Yanchuk, Eckhaus Instability in Systems with Large Delay, Phys. Rev. Lett. 96, 220201 (2006).

[27] M. C. Soriano, J. García-Ojalvo, C. R. Mirasso, and I. Fischer, Complex photonics: Dynamics and applications of delay-coupled semiconductors lasers, Rev. Mod. Phys. 85, 421 (2013).

[28] O. D'Huys, T. Jüngling, and W. Kinzel, Stochastic switching in delay-coupled oscillators, Phys. Rev. E 90, 032918 (2014).

[29] S. Yanchuk and M. Wolfrum, A multiple time scale approach to the stability of external cavity modes in the Lang-Kobayashi system using the limit of large delay, SIAM J. Appl. Dyn. Syst. 9, 519 (2010).

[30] T. Erneux and P. Glorieux, Laser Dynamics (Cambridge University Press, Cambridge, 2010).

[31] K. Engelborghs, T. Luzyanina, and D. Roose, Numerical bifurcation analysis of delay differential equations using DDE-BIFTOOL, ACM Trans. Math. Softw. 28, 1 (2002).

[32] P. Pérez, A. Quirce, A. Valle, A. Consoli, I. Noriega, L. Pesquera, and I. Esquivias, Photonic generation of microwave signals using a single-mode vcsel subject to dual-beam orthogonal optical injection, IEEE Photonics J. 7, 1 (2015).

[33] S.-C. Chan, S.-K. Hwang, and J.-M. Liu, Period-one oscillation for photonic microwave transmission using an optically injected semiconductor laser, Opt. Express 15, 14921 (2007).

[34] S.-C. Chan and J.-M. Liu, Tunable narrow-linewidth photonic microwave generation using semiconductor laser dynamics, IEEE J. Sel. Top. Quantum Electron. 10, 1025 (2004).

[35] S. Yanchuk and G. Giacomelli, Spatio-temporal phenomena in complex systems with time delays, J. Phys. A: Math. Theor. 50, 103001 (2017). 